\title{
Irritable Bowel Syndrome, Particularly the Constipation-Predominant Form, Involves an Increase in Methanobrevibacter smithii, Which Is Associated with Higher Methane Production
}

\author{
Ujjala Ghoshal ${ }^{1}$, Ratnakar Shukla ${ }^{1}$, Deepakshi Srivastava ${ }^{2}$, and Uday C Ghoshal ${ }^{2}$ \\ Departments of ${ }^{1}$ Microbiology and ${ }^{2}$ Gastroenterology, Sanjay Gandhi Postgraduate Institute of Medical Sciences, Lucknow, India
}

Background/Aims: Because Methanobrevibacter smithii produces methane, delaying gut transit, we evaluated $M$. smithii loads in irritable bowel syndrome (IBS) patients and healthy controls $(\mathrm{HC})$. Methods: Quantitative real-time polymerase chain reaction for $M$. smithii was performed on the feces of 47 IBS patients (Rome III) and $30 \mathrm{HC}$. On the lactulose hydrogen breath test (LHBT, done for 25 IBS patients), a fasting methane result $\geq 10 \mathrm{ppm}$ using $10 \mathrm{~g}$ of lactulose defined methane-producers. Results: Of 47, 20 had constipation (IBS-C), 20 had diarrhea (IBS-D) and seven were not sub-typed. The M. smithii copy number was higher among IBS patients than $\mathrm{HC}\left(\log _{10} 5.4\right.$, interquartile range [IQR; 3.2 to 6.3 ] vs 1.9 [0.0 to 3.4], $p<0.001$ ), particularly among IBSC compared to IBS-D patients $\left(\log _{10} 6.1\right.$ [5.5 to 6.6$]$ vs 3.4 [0.6 to 5.7], $p=0.001)$; the copy number negatively correlated with the stool frequency $(R=-0.420, p=0.003)$. The $M$. smithii copy number was higher among methane-producers than nonproducers ( $\log _{10} 6.4$, IQR [5.7 to 7.4 ] vs 4.1 [1.8 to 5.8], $\mathrm{p}=0.001$ ). Using a receiver operating characteristic curve, the best cutoff for $M$. smithii among methane producers was $\log _{10} 6.0$ (sensitivity, $64 \%$; specificity, $86 \%$; area under curve [AUC], 0.896). The AUC for breath methane correlated with the M. smithii copy number among methane producers $(r=0.74, p=0.008)$. Abdominal bloating was more common among methane producers ( $n=9 / 11$ [82\%] vs 5/14 [36\%], $\mathrm{p}=0.021$ ). Conclusions: Patients with IBS, particularly IBS$\mathrm{C}$, had higher copy numbers of M. smithii than HC. On LHBT, breath methane levels correlated with M. smithii loads. (Gut Liver 2016;10:932-938)

Key Words: Methanogenic flora; Real-time polymerase chain reaction; Lactulose hydrogen breath test; Gut transit

\section{INTRODUCTION}

Irritable bowel syndrome (IBS) is a common functional gastrointestinal disorder of unknown aetiology. ${ }^{1,2}$ Several hypotheses have been proposed in pathogenesis of IBS including altered gut microbiota, visceral hypersensitivity, dysmotility, gastrointestinal infection and infestation, dysregulation of brain-gut axis, psychological and genetic factors. ${ }^{2-4}$ Therapeutic manipulation of gut flora using antibiotic or probiotic is known to improve IBS symptoms. ${ }^{5,6}$ Role of gut microbiota and its alterations is being explored in the pathogenesis of this enigmatic disorder. $^{7}$

About $30 \%$ to $62 \%$ of individuals have methane producing bacteria in their gut. ${ }^{8}$ These methanogens are important anaerobic archaea of colonic flora and produce methane by utilizing hydrogen and carbon dioxide. ${ }^{8,9}$ Several in vitro and in vivo studies showed that methane inhibits gastrointestinal motility and therefore, its level may inversely correlate with stool frequency. ${ }^{10,11}$ In addition, area under curve (AUC) for breath methane correlated with severity of constipation among patients with constipation-predominant IBS (IBS-C). ${ }^{11}$ Moreover, treatments with antibiotics targeting methanogens have been shown to improve gut transit and constipation. ${ }^{12-14}$

Culture and molecular studies showed that Methanobrevibacter smithii is the dominant methanogen in the colon and its level varies from undetectable to $10^{10} / \mathrm{g}$ dry weight of stool..$^{15,16}$ Recently, a study reported that breath methane can be measured only when the level of M. smithii exceeds $4.2 \times 10^{5}$ copies per gram of wet stool samples. ${ }^{16}$ However, this study had limitation due to small sample size. ${ }^{15}$ In addition, there is scanty data on relationship between M. smithii and other symptoms of IBS such as abdominal bloating, pain and discomfort. In an earlier

\footnotetext{
Correspondence to: Ujjala Ghoshal

Department of Microbiology, Sanjay Gandhi Postgraduate Institute of Medical Sciences, Raebareli Road, Lucknow 226014, India

Tel: +91-0522-5221, Fax: +91-522-2668129, E-mail: ujjalaghoshal@yahoo.co.in

Received on November 16, 2015. Revised on January 6, 2016. Accepted on January 30, 2016. Published online July 27, 2016

pISSN 1976-2283 eISSN 2005-1212 https://doi.org/10.5009/gnl15588

Ujjala Ghoshal and Ratnakar Shukla contributed equally to this work as first authors.

(a) This is an Open Access article distributed under the terms of the Creative Commons Attribution Non-Commercial License (http://creativecommons.org/licenses/by-nc/4.0) which permits unrestricted non-commercial use, distribution, and reproduction in any medium, provided the original work is properly cited.
} 
case-control study, patients with IBS were less often methane producers than healthy controls (HC); the author suggested that as synthesis of methane from hydrogen reduces the volume of gas, lack of methanogens among patients with IBS might explain occurrence of abdominal bloating among them. ${ }^{17}$ No study, however, tested this hypothesis.

Accordingly, we undertook a study with the following aims: (1) to determine the copy number of $M$. smithii among different subgroups of patients with IBS and HC using quantitative real-time polymerase chain reaction (qPCR); (2) to determine the copy number of $M$. smithii among methane producers and nonproducers; (3) to determine the cutoff level of $M$. smithii among methane producers using receiver operating characteristic (ROC) curve; (4) to correlate the M. smithii load and AUC for breath methane among methane producers; and (5) to study the relationship between different symptoms of IBS and methane production.

\section{MATERIALS AND METHODS}

\section{Patients and controls}

Patients with IBS diagnosed using Rome III criteria ${ }^{18}$ attending the gastroenterology outpatient clinic of a multilevel teaching hospital in northern India were recruited. Patients were classified into three subtypes using Rome III criteria: IBS-C, diarrhea-predominant (IBS-D) and unsubtyped (IBS-U). Patients having organic or metabolic disorders, receiving antibiotics, probiotics or prokinetics, laxatives, drugs altering gastrointestinal motility within previous 4 weeks and those undergoing intestinal surgery in the past were excluded. All the patients were subjected to investigations such as stool microscopy and occult blood testing, hemogram, thyroid function tests and proctosigmoidoscopy. Age and gender matched healthy subjects were included as controls. All HC were apparently healthy and did not have functional gastrointestinal disorders according to Rome III criteria on structured interview. Written informed consent was obtained from each recruited subject. The Institutional Ethics Committee approved the study protocol (PGI/BE/712/2012).

\section{Clinical evaluation}

Each subject was asked to fill up a validated Hindi version of Rome III $^{19}$ questionnaire to record the demographic and clinical symptoms including abdominal bloating or distension, pain/ discomfort, passage of mucus, urgency, straining, feeling of incomplete evacuation, altered bowel habits or stool form, constipation or diarrhoea and type of stool (using Bristol stool chart with pictures as well as descriptors). According to Rome III criteria, type I and II stools were considered to denote constipation, type VI and beyond as diarrhea, and forms changing in days as irregular stool forms. Patient's dietary habit was also recorded in the standard proforma. Patients taking no food of animal origin except milk were classified as vegetarian and those taking foods of animal origin as nonvegetarians.

\section{Sample collection and extraction of DNA}

Three consecutive fresh stool samples were collected from each study participant. Fresh morning stool samples were collected and transported to the laboratory within 1 to 2 hours. Stool sample from each subject was homogenized and divided into three aliquots. All stool samples were stored at $-80^{\circ} \mathrm{C}$ immediately; subsequently, DNA was extracted using the QIAamp Qiagen mini stool kit (QIAGEN, Hilden, Germany) following manufacturer's instructions with some modifications. Concentration of DNA was quantified by NanoDrop ND2000 Spectrophotometer (NanoDrop Products, Wilmington, DE, USA).

\section{Culture condition of $\mathbf{M}$. smithii (American Type Culture Collection-35061, positive controls for qPCR)}

M. smithii (American Type Culture Collection [ATCC]-35061) was cultured in Wilkins-Chalgren media and incubated under anaerobic condition at $37^{\circ} \mathrm{C}$ for 48 hours. This ATCC strain was used as positive controls for qPCR and standard graph was generated. Genomic DNA from ATCC bacterial strain was extracted using heat extraction method and quantified by NanoDrop spectrophotometer. ${ }^{20}$ Standard graph was used to calculate the copy number of 16S rRNA gene for $M$. smithii in stool samples. Standard graph was generated via serial dilution of control DNA (100 ng) to 8 folds (corresponding approximately $10^{1}$ to $10^{8} 16 \mathrm{~S}$ rRNA copies $/ \mu \mathrm{L}$ ). Real-time PCR was performed to estimate the number of copies of 16S rRNA gene of each of serially diluted DNA. Standard graph was used to calculate the efficacy of qPCR using the Rotor gene software (Corbett Research, Sydney, Australia).

\section{5. qPCR}

qPCR was performed in Corbett Research 6000 Q-PCR instrument (Rotor gene 6000 software). The primers used to amplify the M. smithii and universal bacteria are listed in Table $1 .^{21,22}$

Table 1. Primer Sequences for Target Bacterium

\begin{tabular}{|c|c|c|c|c|}
\hline Target bacterium & Primer sequence $\left(5^{\prime}\right.$ to $\left.3^{\prime}\right)$ & Annealing temperature, ${ }^{\circ} \mathrm{C}$ & Amplicon size, bp & Reference \\
\hline \multirow[t]{2}{*}{ Methanobrevibacter smithii } & FP: CCGGGTATCTAATCCGGTTC & 62 & 123 & Dridi et al. ${ }^{2}$ \\
\hline & RP: CTCCCAGGGTAGAGGTGAAA & & & \\
\hline \multirow[t]{2}{*}{ Universal bacteria } & FP: TCCTACGGGAGGCAGCAGTG & 65 & 446 & Lyra et al. ${ }^{22}$ \\
\hline & RP: GGACTACCAGGGTATCTAATCCTGT & & & \\
\hline
\end{tabular}


Each PCR was carried out in a final volume of $25 \mu \mathrm{L}$, comprising of $12.5 \mu \mathrm{L}$ of Power SYBR ${ }^{\circledR}$ Green PCR master mixture (Applied Biosystems, Carlsbad, CA, USA), $0.25 \mu \mathrm{L}$ of 10 pmole of forward and reverse primers and $100 \mathrm{ng}$ of DNA. Each set of reaction was run with positive and negative controls. qPCR was run in duplicate for each sample. The following thermal cycling parameters were used for amplification of DNA: reaction cycle at $95^{\circ} \mathrm{C}$ for 10 minutes to activate the AmpliTaq Gold ${ }^{\circledR}$ DNA polymerase (Applied Biosystems, Carlsbad, CA, USA) followed by 40 cycles of initial denaturation at $95^{\circ} \mathrm{C}$ for 15 seconds, 30 seconds of annealing at optimal temperature (Table 1), elongation at $72^{\circ} \mathrm{C}$ for 30 seconds. The quality of qPCR product was confirmed by melting curve analysis with increasing temperature from $60^{\circ} \mathrm{C}$ to $95^{\circ} \mathrm{C}$ (at regular increment of 0.5 degree for 5 seconds) to confirm that the fluorescence signal was due to desired amplicon and not from primer dimer or nonspecific PCR products. The qPCR amplified amplicon was further confirmed by applying on $2 \%$ agarose gel electrophoresis along with 100 base pair ladder.

\section{Breath test}

Lactulose hydrogen breath test (LHBT) was performed according to a standard protocol. ${ }^{23}$ All study subjects undergoing LHBT were advised to avoid complex carbohydrate rich diets one day before the test. Smoking and physical exercise were not allowed 2 hours before and during the test. Patients washed their mouth with antiseptic mouthwash to avoid any erroneous results due to action of oral bacteria on the test sugar. Breath sample was collected to determine the basal breath methane level (ppm) after a 12 hours of fasting using breath gas analyzer (Breathtracker SC, Digital Microlyzer; Quintron, Milwaukee, WI, USA). An average of 3 readings was taken for fasting breath methane level. Thereafter, patients ingested $15 \mathrm{~mL}$ solution containing 10 g lactulose and level of breath methane was estimated every 15 minutes for a duration of 240 minutes. ${ }^{24}$ Rise in methane level was calculated by subtracting the average basal methane value from the highest value obtained. Fasting breath methane level $\geq 10$ ppm or increase by $\geq 10$ ppm above basal after 10 -g lactulose ingestion was diagnostic criteria of methane-producer. ${ }^{25}$ Total production of methane gas was calculated by AUC. ${ }^{24}$ The person performing the breath test was blinded to the result of fecal microbiota analysis.

\section{Statistical analysis}

SPSS version 15.0 (SPSS Inc., Chicago, IL, USA) and Graph Prism version 5.0 (Graph Pad Software Inc., La Jolla, CA, USA) were used for statistical analysis. The ratio of copy number of 16S rRNA of $M$. smithii to total 16S rRNA copy number of universal bacteria was calculated to find out relative difference. The value of relative difference was transformed into $\log _{10}$ and presented as median and interquartile range (IQR). Categorical and continuous data were analyzed using chi-square and Mann-
Whitney U tests, respectively. Kruskal-Wallis test was used for comparisons of more than two variables. Bonferroni correction was applied for multiple comparisons. Spearman rank correlation was applied to determine the correlation between copy number of $M$. smithii and AUC for breath methane. ROC curve was used to determine the best cutoff level of $M$. smithii to identify methane producers. The $\mathrm{p}$-values $<0.05$ were considered as significant.

\section{RESULTS}

\section{Demographic and clinical parameters of patients with IBS and HC}

The demographic and clinical parameters of patients with IBS and HC are summarized in Table 2. Patients with IBS $(n=47)$ and HC $(n=30)$ were comparable in age (34-year [19 to 68] vs 35 -year [21 to 69], $\mathrm{p}=0.925)$ and gender (39/47 [83\%] vs 22/30 [73\%] male, $p=0.309)$. Twenty patients had IBS-C, 20 IBS-D and remaining seven could not be sub-typed using Rome III criteria.

\section{Quantitative determination of $M$. smithii and its association with clinical symptoms}

M. smithii was detected in 19 of 20 (95\%) patients with IBSC, 15 of 20 (75\%) with IBS-D and 7 of 7 (100\%) with IBSU. Number of copies of $M$. smithii was higher among patients with IBS than HC $\left(\log _{10} 5.4\right.$, IQR [3.2 to 6.3] vs 1.9 [0.0 to 3.4], $\mathrm{p}<0.001$ ) (Fig. 1A). Number of copies of $M$. smithii was higher among patients with IBS-C than HC and IBS-D $\left(\log _{10} 6.1\right.$ [5.5 to 6.6] vs 1.9 [0.0 to 3.4] and $\log _{10} 3.4$ [0.6 to 5.7]; $\mathrm{p}<0.001$ and $\mathrm{p}=0.004$, respectively) (Fig. 1B). Copy number of $M$. smithii inversely correlated with stool frequency among patients with IBS ( $\mathrm{r}=-0.420, \mathrm{p}=0.003$ ) (Fig. 1C). In addition, number of copies of $M$. smithii was lower among IBS patients passing more than 3 stools per day than those without $\left(n=25 / 47, \log _{10} 3.6\right.$, IQR [2.4 to 5.6] vs $22 / 47, \log _{10} 5.9$, IQR [4.9 to 6.6]; $p=0.002$ ). M. smithii load was higher in patients with Bristol stool type 1 to 2 than those with stool type 3 to 5 and type 6 to $7\left(\log _{10} 5.6\right.$ [5.0 to 6.5] vs $\log _{10} 3.4$ [0.6 to 5.9] and $\log _{10} 3.7$ [2.4 to 5.5]; $\mathrm{p}<0.03$ and $\mathrm{p}<0.02$, respectively).

\section{Relationship between $M$. smithii load and breath methane on LHBT}

LHBT was performed among (25/47) patients with IBS (12 patients with IBS-C and 13 with IBS-D). Of 25 patients who underwent LHBT, IBS-C patients were more often methaneproducer than IBS-D (8/12 [67\%] vs 3/13 [23\%], p=0.047). Number of copies of $16 \mathrm{~S}$ rRNA of $M$. smithii was higher among methane-producers than non-producers $\left(\log _{10} 6.4\right.$ [5.7 to 7.4$]$ vs 4.1 [1.8 to 5.8], $\mathrm{p}=0.001$ ) (Fig. 2A). 
Table 2. Demographics and Clinical Symptoms in Patients with IBS and HC

\begin{tabular}{|c|c|c|c|}
\hline Parameter & IBS $(n=47)$ & $\mathrm{HC}(\mathrm{n}=30)$ & p-value \\
\hline Age, yr & $34(19-68)$ & $35(21-69)$ & $0.925^{*}$ \\
\hline Male sex & $39(83)$ & $22(73)$ & 0.309 \\
\hline \multicolumn{4}{|l|}{ Predominant bowel habits (Rome III criteria) } \\
\hline IBS-C & $20(43)$ & - & - \\
\hline IBS-D & $20(43)$ & - & - \\
\hline IBS-U & $7(14)$ & - & - \\
\hline \multicolumn{4}{|l|}{ Clinical symptoms } \\
\hline Visible abdominal distension & $34(72)$ & $2(7)$ & $<0.001$ \\
\hline Abdominal bloating/feeling of abdominal distension $>1 / 4$ of days & $22(47)$ & 0 & $<0.001$ \\
\hline Abdominal pain & $35(74)$ & $1(3)$ & $<0.001$ \\
\hline Abdominal discomfort & $47(100)$ & $2(7)$ & $<0.001$ \\
\hline Relief of pain/discomfort with bowel movement & $46(98)$ & $2(7)$ & $<0.001$ \\
\hline More frequent stool at onset of pain & $13(28)$ & 0 & 0.001 \\
\hline Loose stool at onset of pain & $14(30)$ & 0 & 0.001 \\
\hline Urgency & $17(36)$ & 0 & $<0.001$ \\
\hline Passage of mucus & $35(74)$ & $1(3)$ & $<0.001$ \\
\hline Passage of mucus $>1 / 4$ of defecation & $14(28)$ & 0 & 0.001 \\
\hline Feeling of incomplete evacuation & $45(96)$ & $3(10)$ & $<0.001$ \\
\hline Feeling of incomplete evacuation $>1 / 4$ of defecation & $29(62)$ & 0 & $<0.001$ \\
\hline Straining during defecation & $27(57)$ & $1(3)$ & $<0.001$ \\
\hline Irregular stool form & $33(70)$ & $1(3)$ & $<0.001$ \\
\hline Irregular stool frequency & $39(83)$ & $1(3)$ & $<0.001$ \\
\hline \multicolumn{4}{|l|}{ Diet } \\
\hline Vegetarian & $14(30)$ & $12(40)$ & NS \\
\hline Nonvegetarian & $33(70)$ & $18(60)$ & NS \\
\hline
\end{tabular}

Data are presented as median (range) or number (\%).

IBS, irritable bowel syndrome; HC, healthy controls; IBS-C, constipation-predominant IBS; IBS-D, diarrhea-predominant IBS; IBS-U, unsubtyped IBS; NS, not significant.

*Nonparametric independent sample test. A chi-square test was used for all other categorical data. $\mathrm{p}<0.05$ was considered significant.

A

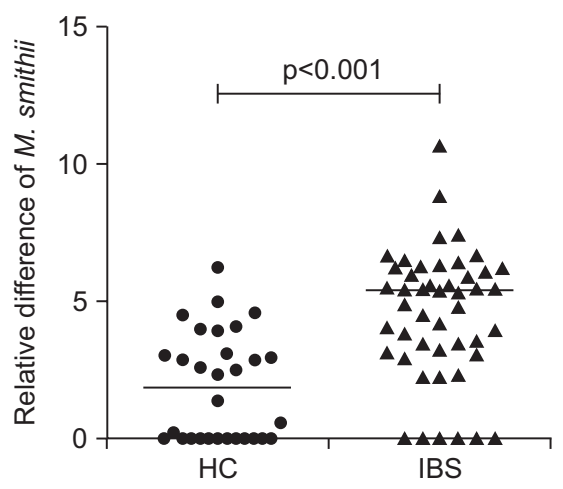

B

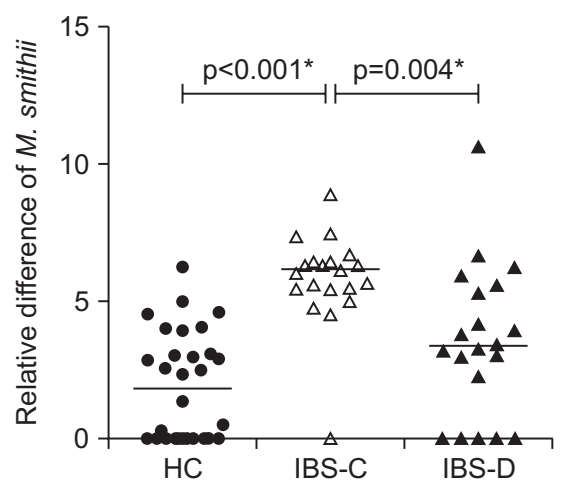

C

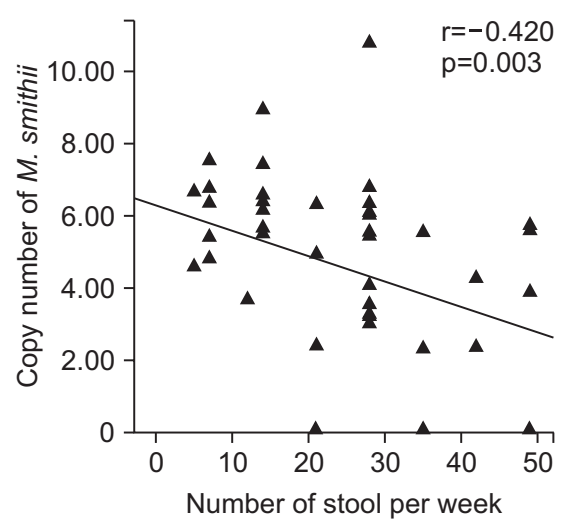

Fig. 1. (A) Copy numbers of Methanobrevibacter smithii among patients with irritable bowel syndrome (IBS) and healthy controls (HC). (B) Copy numbers of M. smithii among patients with constipation-predominant IBS (IBS-C), diarrhea-predominant IBS (IBS-D), and HC. (C) Correlation between copy numbers of $M$. smithii and number of stools per week. $\mathrm{p}<0.05$ was considered as significant. *Bonferroni corrected $\mathrm{p}$-value. 
A

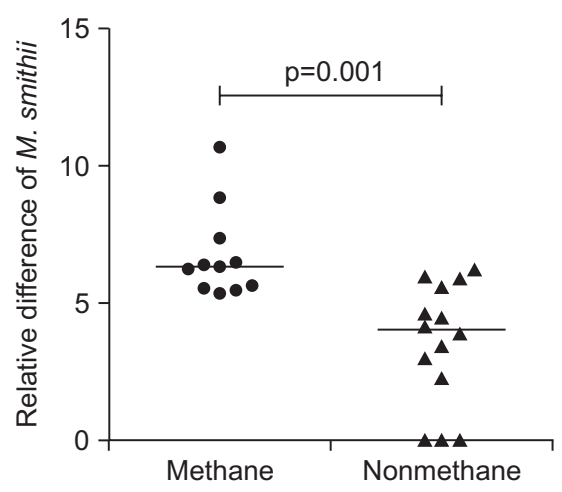

B

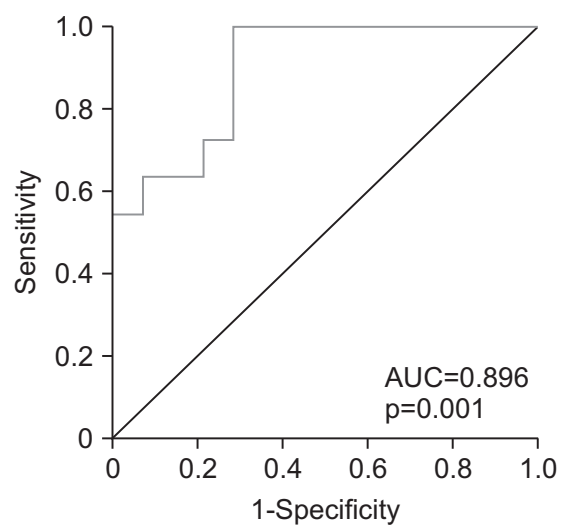

C

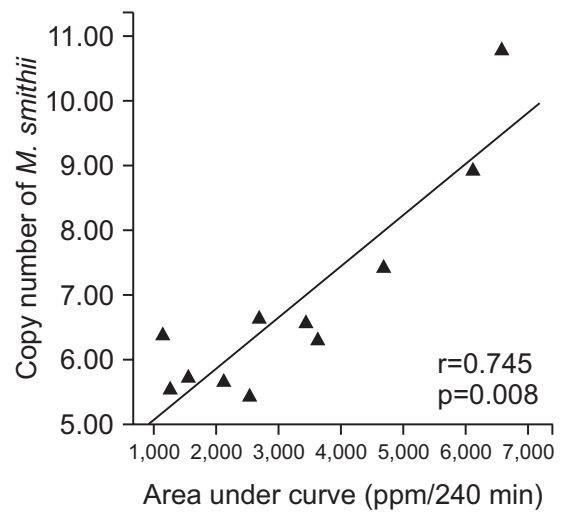

Fig. 2. (A) Copy numbers of Methanobrevibacter smithii among methane producers and nonproducers. (B) Receiver operating characteristic curve showing the area under the curve (AUC). (C) Correlation between copy numbers of M. smithii and the AUC. $p<0.05$ was considered as significant.

Table 3. Relationships between Different Symptoms of Irritable Bowel Syndrome among Methane Producers and Methane Nonproducers

\begin{tabular}{lccc}
\hline \multicolumn{1}{c}{ Symptom } & Methane producer (n=11) & Methane nonproducer (n=14) & $\mathrm{p}$-value \\
\hline Abdominal bloating & $9(82)$ & $5(36)$ & $11(79)$ \\
Abdominal pain & $9(82)$ & $14(100)$ & NS \\
Relief of pain or discomfort with bowel movement & $11(100)$ & $7(50)$ & NS \\
Urgency & $2(18)$ & $9(64)$ & NS \\
Passage of mucus per rectum & $7(64)$ & $8(57)$ & NS \\
Feeling of incomplete evacuation $>1 / 4$ of defecation & $9(82)$ & $5(36)$ & NS \\
Straining & $7(64)$ & $12(86)$ & NS \\
Irregular stool form & $7(64)$ & $12(86)$ & NS \\
Irregular stool frequency & $8(73)$ & $9(64)$ & 0.005 \\
More frequent stool at onset of pain & $1(9)$ & $8(57)$ & 0.013 \\
Loose stool at onset of pain & $1(9)$ &
\end{tabular}

Data are presented as number (\%).

NS, not significant $(p>0.05)$.

\section{Cutoff level of $M$. smithii among methane producers on LHBT}

Using ROC curve, an optimal cutoff of M. smithii among methane producers on LHBT was $\log _{10} 6.0$ (area under ROC curve, 0.896 [95\% CI, 0.77 to $1.00 ; p=0.001]$ ), which had sensitivity and specificity of 64\% and 86\%, respectively (Fig. 2B).

\section{Correlation between M. smithii load and AUC for breath methane}

AUC for breath methane correlated positively with number of copies of M. smithii among methane producers (Spearman correlation coefficient, $r=0.745 ; \mathrm{p}=0.008$ ) (Fig. 2C).

\section{Relationship between different symptoms of IBS and methane production}

Abdominal bloating was more common among methane producers than nonproducers $(n=9 / 11$ [82\%] vs 5/14 [36\%], $\mathrm{p}=0.021)$. Methane producers more often had Bristol stool type 1 to 2; in contrast, the methane nonproducers had stool type 6 to 7 ( $\mathrm{n}=8 / 12$ [66\%] vs 10/13 [77\%], $\mathrm{p}=0.047)$. Other symptoms like abdominal pain, relief of pain or discomfort with bowel movement, urgency, passage of mucus with stool, feeling of incomplete evacuation, straining, irregular stool form and frequency were comparable among methane producers and nonproducers (Table 3).

\section{DISCUSSION}

The present study shows that (1) number of copies $M$. smithii was higher among patients with IBS, particularly IBS-C than IBS-D and HC; (2) M. smithii load inversely correlated with stool frequency among patients with IBS; (3) number of copies of $M$. smithii was less among patients with IBS having more than 3 stool per day than those without; (4) number of copies of M. smithii was higher among methane producers than nonproducers; (5) threshold value of M. smithii among LHBT positive methane producers was $\log _{10} 6.0$; (6) AUC for breath methane 
correlated positively with load of $M$. smithii among methane producers; and (7) methane producers more often had abdominal bloating than nonproducers.

Human colon harbors diverse microbial species, which produces methane. ${ }^{26}$ Ten percent of total anaerobes residing in human colon are methanogens and $80 \%$ to $100 \%$ of which is M. smithii by PCR. ${ }^{26}$ There are limited data on M. smithii load among patients with IBS using qPCR. One study by Kim et al. ${ }^{15}$ showed that $M$. smithii was predominant methanogen among patients with IBS-C, which is in accordance with our study. In contrast, another study showed that the number of copies of M. smithii was lower among patients with IBS than controls. ${ }^{27}$ Therefore, more studies are necessary to determine whether higher level of M. smithii is associated with IBS-C. The present study provides further evidence of relationship between $M$. smithii and IBS-C.

We wish to emphasize that the load of M. smithii may be more important in pathogenesis of IBS, particularly IBS-C than mere detection of it. ${ }^{15}$ Using qPCR, we found that optimal cutoff level of M. smithii in stool sample was $\log _{10} 6$ to detect methane in breath on LHBT. In a previous study, the author used culture method and found that minimum level of methanogen must be $2 \times 10^{8}$ per gram of dry weight of stool to detect significant amount of methane in breath (>6 ppm). ${ }^{28} \mathrm{M}$. smithii was identified using morphological, physiological, and immunological methods. Since, culture method can detect only live bacteria and M. smithii, as anaerobes, could die due to exposure of air. ${ }^{28}$ qPCR is expected to be more efficient and sensitive tool for identification of colonic methanogens in stool samples.

Patients with IBS producing methane in breath on LHBT had abdominal bloating more often. These results were consistent with the previous study showing that methane production was associated with abdominal bloating, pain and flatulence ${ }^{26}$ and contradicts the hypothesis suggested in another study claiming that methanogenesis might prevent abdominal bloating by reducing the volume of the gas while synthesis of methane from four atoms of hydrogen and one atom of cardon. ${ }^{17}$ However, this was merely a hypothesis and abdominal bloating was not assessed in that study. ${ }^{17}$ Our results reject this hypothesis. This is quite expected as abdominal bloating not only depends on volume of gas inside the lumen of the gut but its preferential retention within the small bowel, gut motility, visceral sensation and regularity and completeness of defecation..$^{26,29,30}$

In this study, number of copies of $M$. smithii was higher among methane producer than nonproducer suggesting that $M$. smithii was responsible for producing methane on LHBT. This finding is consistent with a recent study showing that $M$. smithii load was higher among methane producers than nonproducers. ${ }^{15}$ Evidence from the previous studies showed that higher level of breath methane production correlated with severity of constipation. ${ }^{11}$ However, source of methane producing bacterium among patients with IBS was not clearly understood. Therefore, our study supports the previous hypothesis ${ }^{15}$ that $M$. smithii could be considered as an important methanogen in stool sample responsible for breath methane production among patients with IBS. The number of copies of M. smithii inversely correlated with stool frequency among patients with IBS. Therefore, higher level of M. smithii could be considered as predisposing factor for the development of constipation.

Elimination of methanogenic flora using antibiotic treatment may contribute to therapeutic benefits at least in a subgroup of patients with IBS (IBS-C). ${ }^{13}$ In a study of 111 Rome I IBS subjects, neomycin improved the constipation severity than placebo $(44.0 \% \pm 12.3 \%$ vs $5.0 \% \pm 5.1 \%, \mathrm{p}<0.05)$ among constipation-predominant methane producers. ${ }^{13}$ In a case report, a patient with slow transit constipation associated with excess methane production improved after treatment with rifaximin, which reduced breath methane level, accelerated colonic transit and changed stool form and frequency. ${ }^{12}$ Moreover, mixture of rifaximin and neomycin was more effective in relieving the methane production and constipation symptoms (87\% and 85\%) than neomycin (33\% and 63\%) or rifaximin alone (28\% and 56\%) among methane producing IBS patients. ${ }^{31}$

In conclusion, number of copies of $M$. smithii was higher among patients with IBS, particularly in IBS-C than HC and IBS-D. Moreover, number of copies of $M$. smithii was higher among methane producers than nonproducers. Best cutoff level of $M$. smithii among LHBT positive methane producers was $\log _{10} 6.0$. AUC for breath methane had correlation with load of M. smithii among methane producers. Abdominal bloating was more common among methane producers than nonproducers.

\section{CONFLICTS OF INTEREST}

No potential conflict of interest relevant to this article was reported.

\section{ACKNOWLEDGEMENTS}

The authors wish to thank all the recruited subjects and volunteers for taking part in this study. Ratnakar Shukla thanks the Department of Science and Technology for providing his fellowship.

\section{REFERENCES}

1. Longstreth GF, Thompson WG, Chey WD, Houghton LA, Mearin F, Spiller RC. Functional bowel disorders. Gastroenterology 2006; 130:1480-1491.

2. Ghoshal UC, Shukla R, Ghoshal U, Gwee KA, Ng SC, Quigley EM. The gut microbiota and irritable bowel syndrome: friend or foe? Int J Inflam 2012;2012:151085.

3. Parkes GC, Brostoff J, Whelan K, Sanderson JD. Gastrointestinal microbiota in irritable bowel syndrome: their role in its pathogen- 
esis and treatment. Am J Gastroenterol 2008;103:1557-1567.

4. Ohman L, Simrén M. New insights into the pathogenesis and pathophysiology of irritable bowel syndrome. Dig Liver Dis 2007; 39:201-215.

5. Pimentel M, Lembo A, Chey WD, et al. Rifaximin therapy for patients with irritable bowel syndrome without constipation. N Engl J Med 2011;364:22-32.

6. Cash BD. Emerging role of probiotics and antimicrobials in the management of irritable bowel syndrome. Curr Med Res Opin 2014;30:1405-1415.

7. Shukla R, Ghoshal U, Dhole TN, Ghoshal UC. Fecal microbiota in patients with irritable bowel syndrome compared with healthy controls using real-time polymerase chain reaction: an evidence of dysbiosis. Dig Dis Sci 2015;60:2953-2962.

8. Attaluri A, Jackson M, Valestin J, Rao SS. Methanogenic flora is associated with altered colonic transit but not stool characteristics in constipation without IBS. Am J Gastroenterol 2010;105:14071411.

9. Rana SV, Sinha SK, Sharma S, Kaur H, Bhasin DK, Singh K. Effect of predominant methanogenic flora on outcome of lactose hydrogen breath test in controls and irritable bowel syndrome patients of north India. Dig Dis Sci 2009;54:1550-1554.

10. Pimentel M, Lin HC, Enayati P, et al. Methane, a gas produced by enteric bacteria, slows intestinal transit and augments small intestinal contractile activity. Am J Physiol Gastrointest Liver Physiol 2006;290:G1089-G1095.

11. Chatterjee S, Park S, Low K, Kong Y, Pimentel M. The degree of breath methane production in IBS correlates with the severity of constipation. Am J Gastroenterol 2007;102:837-841.

12. Ghoshal UC, Srivastava D, Verma A, Misra A. Slow transit constipation associated with excess methane production and its improvement following rifaximin therapy: a case report. J Neurogastroenterol Motil 2011;17:185-188.

13. Pimentel M, Chatterjee S, Chow EJ, Park S, Kong Y. Neomycin improves constipation-predominant irritable bowel syndrome in a fashion that is dependent on the presence of methane gas: subanalysis of a double-blind randomized controlled study. Dig Dis Sci 2006;51:1297-1301.

14. Ghoshal UC, Srivastava D, Misra A. Sa1378 Reduction of breath methane using rifaximin shortens colon transit time and improves constipation: a randomized double-blind placebo controlled trial. Gastroenterology 2015;148:4 Suppl 1:S308-S309.

15. Kim G, Deepinder F, Morales W, et al. Methanobrevibacter smithii is the predominant methanogen in patients with constipation-predominant IBS and methane on breath. Dig Dis Sci 2012;57:32133218.

16. Bajaj JS, Gillevet PM, Hylemon PB. Methanogenesis in irritable bowel syndrome: a lot of hot air? Dig Dis Sci 2012;57:3045-3046.

17. Rana SV, Sharma S, Sinha SK, Kaur H, Sikander A, Singh K. Incidence of predominant methanogenic flora in irritable bowel syndrome patients and apparently healthy controls from North India.
Dig Dis Sci 2009;54:132-135.

18. Hattori T, Fukudo S. Use of Rome III criteria for diagnosing irritable bowel syndrome. Nihon Rinsho 2006;64:1425-1428.

19. Ghoshal UC, Gwee KA, Chen M, et al. Development, translation and validation of enhanced Asian Rome III questionnaires for diagnosis of functional bowel diseases in major Asian languages: a Rome Foundation-Asian Neurogastroenterology and Motility Association Working Team report. J Neurogastroenterol Motil 2015;21:83-92.

20. Bartosch S, Fite A, Macfarlane GT, McMurdo ME. Characterization of bacterial communities in feces from healthy elderly volunteers and hospitalized elderly patients by using real-time PCR and effects of antibiotic treatment on the fecal microbiota. Appl Environ Microbiol 2004;70:3575-3581.

21. Dridi B, Henry M, El Khéchine A, Raoult D, Drancourt M. High prevalence of Methanobrevibacter smithii and Methanosphaera stadtmanae detected in the human gut using an improved DNA detection protocol. PLoS One 2009;4:e7063.

22. Lyra A, Rinttilä T, Nikkilä J, et al. Diarrhoea-predominant irritable bowel syndrome distinguishable by 16S rRNA gene phylotype quantification. World J Gastroenterol 2009;15:5936-5945.

23. Ghoshal UC. How to interpret hydrogen breath tests. J Neurogastroenterol Motil 2011;17:312-317.

24. Ghoshal UC, Srivastava D, Ghoshal U, Misra A. Breath tests in the diagnosis of small intestinal bacterial overgrowth in patients with irritable bowel syndrome in comparison with quantitative upper gut aspirate culture. Eur J Gastroenterol Hepatol 2014;26:753-760.

25. Jang SI, Kim JH, Youn YH, Park H, Lee SI, Conklin JL. Relationship between intestinal gas and the development of right colonic diverticula. J Neurogastroenterol Motil 2010;16:418-423.

26. Triantafyllou K, Chang C, Pimentel M. Methanogens, methane and gastrointestinal motility. J Neurogastroenterol Motil 2014;20:3140.

27. Rajilić-Stojanović M, Biagi E, Heilig HG, et al. Global and deep molecular analysis of microbiota signatures in fecal samples from patients with irritable bowel syndrome. Gastroenterology 2011;141:1792-1801.

28. Weaver GA, Krause JA, Miller TL, Wolin MJ. Incidence of methanogenic bacteria in a sigmoidoscopy population: an association of methanogenic bacteria and diverticulosis. Gut 1986;27:698-704.

29. Agrawal A, Whorwell PJ. Review article: abdominal bloating and distension in functional gastrointestinal disorders: epidemiology and exploration of possible mechanisms. Aliment Pharmacol Ther 2008;27:2-10.

30. Lacy BE, Gabbard SL, Crowell MD. Pathophysiology, evaluation, and treatment of bloating: hope, hype, or hot air? Gastroenterol Hepatol (N Y) 2011;7:729-739.

31. Low K, Hwang L, Hua J, Zhu A, Morales W, Pimentel M. A combination of rifaximin and neomycin is most effective in treating irritable bowel syndrome patients with methane on lactulose breath test. J Clin Gastroenterol 2010;44:547-550. 